\title{
Abalone Muscle Texture Evaluation and Prediction Based on TPA Experiment
}

\author{
Jiaxu Dong, ${ }^{1,2}$ Xiaoyan Fang, ${ }^{1,2}$ Huihui Wang, ${ }^{1,2}$ Xu Zhang, ${ }^{1,2}$ and Xueheng Tao ${ }^{1,2}$ \\ ${ }^{1}$ Key Laboratory for Seafood Processing Technology and Equipment of Liaoning Province, Dalian Polytechnic University, \\ Dalian 116034, China \\ ${ }^{2}$ National Engineering Research Center of Seafood, Dalian 116034, China
}

Correspondence should be addressed to Xueheng Tao; taohouse@163.com

Received 14 April 2017; Revised 21 July 2017; Accepted 7 August 2017; Published 20 September 2017

Academic Editor: Alessandro Genovese

Copyright (C) 2017 Jiaxu Dong et al. This is an open access article distributed under the Creative Commons Attribution License, which permits unrestricted use, distribution, and reproduction in any medium, provided the original work is properly cited.

\begin{abstract}
The effects of different heat treatments on abalones' texture properties and sensory characteristics were studied. Thermal processing of abalone muscle was analyzed to determine the optimal heat treatment condition based on fuzzy evaluation. The results showed that heat treatment at $85^{\circ} \mathrm{C}$ for 1 hour had certain desirable effects on the properties of the abalone meat. Specifically, a back propagation (BP) neural network was introduced to predict the equations of statistically significant sensory hardness, springiness, and smell using the texture data gained through TPA (texture profile analysis) experiments as input and sensory evaluation data as the desired output. The final outcome was that the predictability was proved to be satisfactory, with an average error of $6.93 \%$.
\end{abstract}

\section{Introduction}

Abalone, a kind of edible sea snail, is a highly prized and expensive ingredient in Chinese cuisine. The most common variety is Haliotis discus hannai Ino, found in northern China [1]. The species, which contains protein, fat, vitamins, glycogen, and many other trace elements, has high nutritional value and is widely used in healthcare $[2,3]$. With the recent improvements in living standards, abalone represents an increasingly large proportion of individuals' dietary structure, leading to increasing demand for its texture. Under thermal processing conditions, we should be able to effectively extend the storage period for abalone processing [4].

At present, research into the heat treatment of abalone meat is mainly focused on the sensory evaluation and texture analyzer evaluation methods. The former, which involves the use of human sensory organs, is a relatively accurate texture evaluation method. However, the application of abalone in sensory evaluation is time-consuming, laborious, and prone to errors and omissions. The TPA experimental method is used to evaluate the physical or mechanical indicators of food via a texture analyzer that establishes the relationship between the test signal and the texture parameters to analyze the food texture. Although the method is fairly objective, the result is far removed from expectations because of the different structures of instrumental and human masticatory organs.

In recent years, with the rapid development of electronic technology, increasing numbers of researchers are beginning to apply electronic sensing technology to the texture analysis of food, including electromyography and the piezoelectricfilm sensor [5]. At the same time, many kinds of bionic equipment have been emerging, such as the electronic tongue, which are able to simulate human perception methods for testing [6]. However, these methods are expensive and complicated and therefore do not align with the requirements of enterprise.

Instead, for this paper, the texture parameters of springiness, hardness, chewiness, and resilience of abalone were measured using a texture analyzer. The effects of different heat treatment conditions on abalones' texture properties and sensory characteristics were analyzed. To attain the optimal condition, the researcher used the method of setting up a fuzzy matrix. In addition, an objective, fast, and accurate evaluation model for abalone quality was designed, based on the $\mathrm{BP}$ artificial network. 
TABLE 1: Factors and levels table for experimental design.

\begin{tabular}{lcc}
\hline $\mathrm{S} / \mathrm{N}$ & $T /^{\circ} \mathrm{C}$ & $t / \mathrm{min}$ \\
\hline 1 & 60 & 15 \\
2 & 60 & 30 \\
3 & 60 & 60 \\
4 & 60 & 120 \\
5 & 60 & 240 \\
6 & 60 & 360 \\
7 & 80 & 15 \\
8 & 80 & 30 \\
9 & 80 & 60 \\
10 & 80 & 120 \\
11 & 80 & 240 \\
12 & 80 & 360 \\
13 & 85 & 15 \\
14 & 85 & 30 \\
15 & 85 & 60 \\
16 & 85 & 120 \\
17 & 85 & 240 \\
18 & 85 & 360 \\
19 & 90 & 15 \\
20 & 100 & 30 \\
21 & 90 & 60 \\
22 & 90 & 120 \\
23 & 100 & 240 \\
24 & 90 & 360 \\
25 & 90 & 120 \\
26 & 900 \\
27 & 90 \\
28 & 90 \\
29 & 90 \\
30 & 60 \\
\hline
\end{tabular}

\section{Materials and Methods}

2.1. Experimental Materials. Fresh abalones (Haliotis discus hannai Ino) were purchased from the Dalian seafood market, weighing $70 \pm 5 \mathrm{~g}$ (including shell) and measuring $8 \pm 1 \mathrm{~cm}$ in length. Similar-sized specimens were selected and immediately sent to the laboratory. After the shells and viscera were removed, the abalone meat was kept in icy water prior to the experiments [4].

2.2. Abalone Treatment. The abalone meat was divided into 5 groups for the experiment, each of which would be subjected to a different processing temperature. The meat was set to the power of a $1500 \mathrm{~W}$ thermostat in a water bath $(\mathrm{HH}-$ 4 Changzhou Rui Zhibo equipment manufacture Co. Ltd.) and conducted at $60,80,85,90$, and $100^{\circ} \mathrm{C}$ for $15,30,60$, 120,240 , and $360 \mathrm{~min}$, respectively [7]. The processing of the meat in the water bath is shown in Figure 1. In addition, an experiment table containing 30 sets of experiments is shown in Table 1.

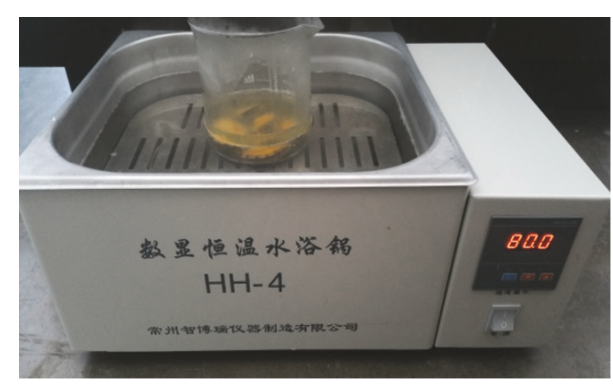

FIGURE 1: The thermal processing of abalone meat.

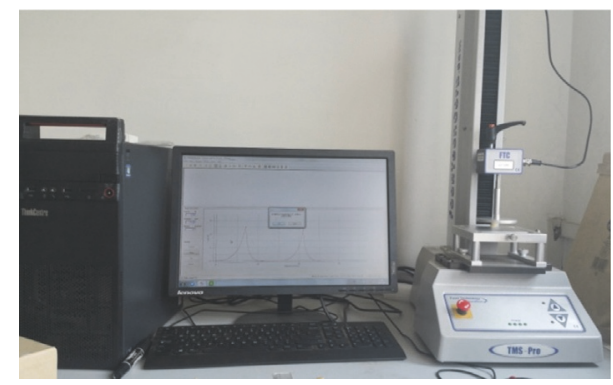

Figure 2: The texture analyzer.

2.3. Texture Profile Analysis (TPA). Following the heat treatments, the abalone meat was cooled at room temperature for $20 \mathrm{~min}$ before starting the experiments. Cylindrical samples of $1.2 \mathrm{~cm}$ (diameter) $\times 1 \mathrm{~cm}$ (height) were cut from the middle of the meat for textural analyses [1]. The upper surface of the sample was the stress surface, which is perpendicular to the muscle fiber. Whole abalone meat was packed in plastic bags, and each sample was set with 5 replicate samples. Using the effect of the height of the sample on the process of sample preparation, the data for the highest and lowest were abandoned and the other data were averaged as the final result of the TPA parameters.

The texture properties of the abalone meat were measured using a texture analyzer (FTC, Virginia, USA), fitted with a $\mathrm{P} / 100$ probe. The samples were placed on the texture analyzer according to their number. Each sample underwent 2 cycles of compression analysis with a compression level of $75 \%$ (relative to sample height), with a $60 \mathrm{~mm} / \mathrm{min}$ test speed and $0.1 \mathrm{~N}$ trigger stress [7]. TPA parameters were evaluated by analyzing the force-displacement curve, including hardness $(\mathrm{HN})$, springiness $(\mathrm{SN})$, chewiness $(\mathrm{CW})$, and resilience (RN). Figure 2 shows the texture analyzer used for the experiment.

\subsection{Sensory Fuzzy Comprehensive Evaluation}

\subsubsection{Establishing the Evaluation System}

(1) Establishing Factor Set $U$. The factor set, a set of indicators that affect the quality of the subject being judged, is expressed as $U=\left(u_{1}, u_{2}, \ldots, u_{m}\right)$. When people chew abalone, its springiness, hardness, and smell are the most direct sensory indicators with which they evaluate it. In this paper, the factor 
TABLE 2: Sensory index criterion of abalone meat.

\begin{tabular}{lccccc}
\hline Sensory score & 5 & 4 & 3 & 2 & Bad \\
\hline Springiness & Very good & Good & Moderate & Very bad \\
Hardness & Moderate & A little hard & Hard & A little soft & Soft \\
Smell & Rich aroma & Moderate aroma & A little aroma & No aroma & Fishy smell \\
\hline
\end{tabular}

collection, composed of three indicators, was identified as $U$ $=\left(\right.$ springiness $u_{1}$, hardness $u_{2}$, smell $\left.u_{3}\right)$.

(2) Establishing Evaluating Index Set $V$. The evaluation set is the collection of quality levels of the evaluated object, which is expressed as $V=\left(v_{1}, v_{2}, \ldots, v_{i}, \ldots, v_{m}\right)(m$ are positive integers with $2<m<8)$ [8]. $V$ is the evaluation set and $v_{i}$ is the evaluation of grade $i$. In this experiment, $m=5$ was selected, which established the evaluation set of the abalone as $V=\left(v_{1}, v_{2}, v_{3}, v_{4}, v_{5}\right) . v_{1}$ is 1 point, representing very bad; $v_{2}$ is 2 points, representing bad; $v_{3}$ is 3 points, representing moderate; $v_{4}$ is 4 points, representing good; $v_{5}$ is 5 points, representing very good.

2.4.2. Sensory Evaluation of Abalone. A total of 30 people were invited to form a sensory evaluation team. The oral health status and sensory evaluation interest of the participants were discerned via a questionnaire. Finally, 11 team members consisting of 6 men and 5 women with good distinctions and descriptive abilities were selected for training through a detailed test, including 4 elderly people with an average age of 65,4 middle-aged men with an average age of 38 , and 3 adolescents.

The sensory evaluation was conducted by 11 selected members from the sensory evaluation team, using the double-blind method [9]. The sensory hardness, sensory springiness, and smell of the abalone meat were evaluated on a 5-point scale. The sensory evaluation index criterion is shown in Table 2.

The evaluation team evaluated the sensory parameters of abalone, and the results were processed using the normalization method. Finally, the matrix $R$ (fuzzy relation matrix) was obtained [8].

2.4.3. Establishing Factor Weight Set a. According to their evaluation experience, 5 professional teachers were invited to state the membership degree that the impact of the 3 indicators had on sensory evaluation. The membership degree was limited to a range from 0 to 1 , before normalization processing was carried out [10]. We could calculate the average value as a power vector in a fuzzy comprehensive evaluation, represented as $a=\left(a_{1}, a_{2}, \ldots, a_{i}, \ldots, a_{m}\right)(m$ represents the number of evaluation indicators).

2.4.4. Establishing the Fuzzy Comprehensive Evaluation Model. The fuzzy matrix composite operator is the key to establishing a comprehensive evaluation model that contains 4 of the most important basic operations. For this paper, the best operator of fuzzy transformation was selected $M(\cdot, \dot{+})$ [11].
The formula for the calculation was as follows:

$$
b_{j}=\sum_{i=1}^{p} a_{i} r_{i j} \quad(j=1,2,3, \ldots, m),
$$

where

$$
\begin{aligned}
& j \text { is sample evaluation grade, } \\
& m \text { is number of sample evaluation grades, } \\
& b_{j} \text { is fuzzy synthesis evaluation result, vector } b, \\
& p \text { is number of evaluation indicators, } \\
& r_{i j} \text { are elements of column } j \text { of line } i \text { of matrix, } \\
& a_{j} \text { is the membership of the first } i \text { element of weight } \\
& \text { vector } a \text {. }
\end{aligned}
$$

2.4.5. Optimization of Heating Conditions. The row vector $b_{j}$ and column vector $v_{i}$ were multiplied to calculate sensory evaluation scores, marked as $A$. The evaluation value of $A$ as the heat treatment condition was analyzed to determine the optimum thermal processing condition for abalone meat.

The scoring formula was

$$
A_{j}=\sum_{i=1}^{m} v_{i} b_{j} \quad(j=1,2,3, \ldots, 20, m) .
$$

By comparing the evaluation values $\left(A_{j}\right)$ of 30 heat treatment conditions, the optimal heat treatment condition was determined.

2.5. Neural Network Analysis. The ANN (artificial neural network) is an information processing system modeled on the human brain's structure and function. More than 40 types of neural network model are used frequently, which can be classified as the Hopfield network, BP neural network, RBF network, and so on [12]. The BP neural network, a kind of widely used forward neural network, contains many layers of networks, including input, concealing, and output layers.

2.5.1. Establishing the Texture Evaluation Model. This network was used to establish the prediction equations of sensory parameters, with the texture data by TPA experiments as the input and sensory evaluation data as the desired output. The TPA parameters of springiness, hardness, chewiness, and resilience were selected as the input layer neurons, while the sensory evaluation parameters of sensory hardness, sensory springiness, and smell were the output layer neurons.

The parameters of the training model were as follows: the allowable error was $1 \times 10^{-6}$, the learning rate was around 0.01 , the dynamic constant was roughly 0.8 , and the maximum number of iterations was approximately 1000 [13]. 


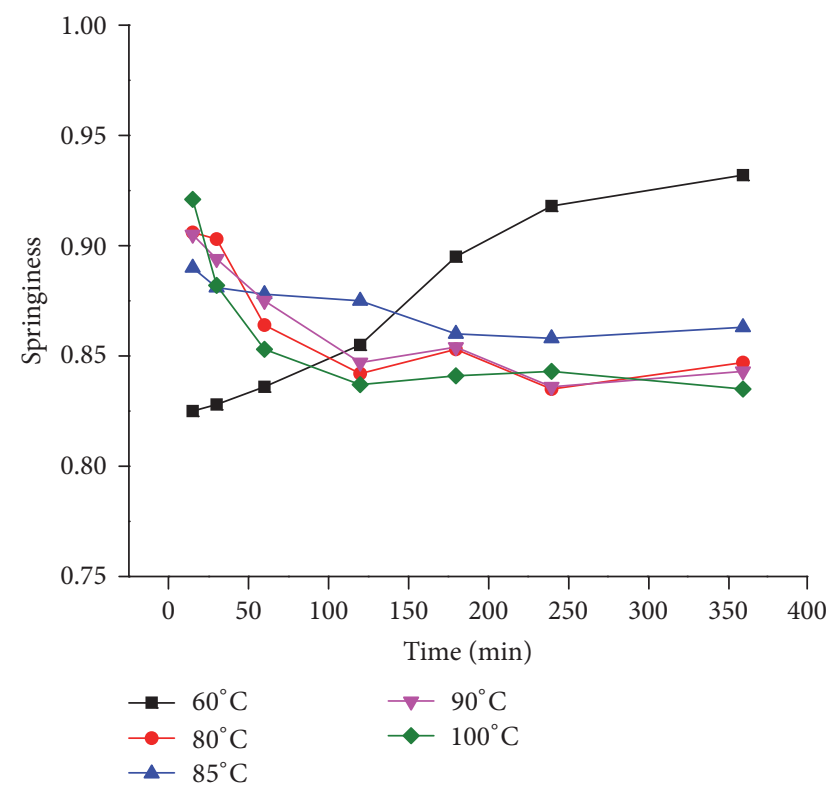

FIgURE 3: Springiness changes during thermal processing.

2.5.2. Validation of Predictive Texture Model. The 12 groups of abalone of nontraining set were randomly evaluated using the constructed neural networks, while being compared with their sensory evaluation results to analyze the rationality of the neural network.

\section{Results and Analysis}

\subsection{Effects of Different Heating Conditions on Abalone Texture}

3.1.1. Effects on Springiness. Figure 3 shows the change to the abalone meat's springiness under different heat treatments. The springiness continued to increase after cooking at $60^{\circ} \mathrm{C}$ before $120 \mathrm{~min}$, with a slight increase from 120 to $360 \mathrm{~min}$. A sharp reduction was observed in meat treated at 80,90 , and $100^{\circ} \mathrm{C}$ before $120 \mathrm{~min}$, but no significant decrease was seen after $120 \mathrm{~min}$. However, similar results were not observed at $85^{\circ} \mathrm{C}$, at which the springiness had not significantly decreased.

3.1.2. Effects on Hardness. Hardness is another textural property of great importance because it can help determine the extent to which consumers accept abalone meat. The change in hardness over time under different heating treatments is shown in Figure 4. The hardness continued to decrease after cooking at $60,80,85,90$, and $100^{\circ} \mathrm{C}$ before $120 \mathrm{~min}$, with the most obvious change seen at $100^{\circ} \mathrm{C}$. In addition, it was found that the higher the heating temperature, the more obvious the tendency to reduce the hardness. A slow reduction in hardness was observed in abalone meat treated at $80,85,90$, and $100^{\circ} \mathrm{C}$ from 120 to $360 \mathrm{~min}$, but a different result was seen at $60^{\circ} \mathrm{C}$. At that temperature, a clear increase in hardness was seen, which was enhanced to the maximum level at $360 \mathrm{~min}$.

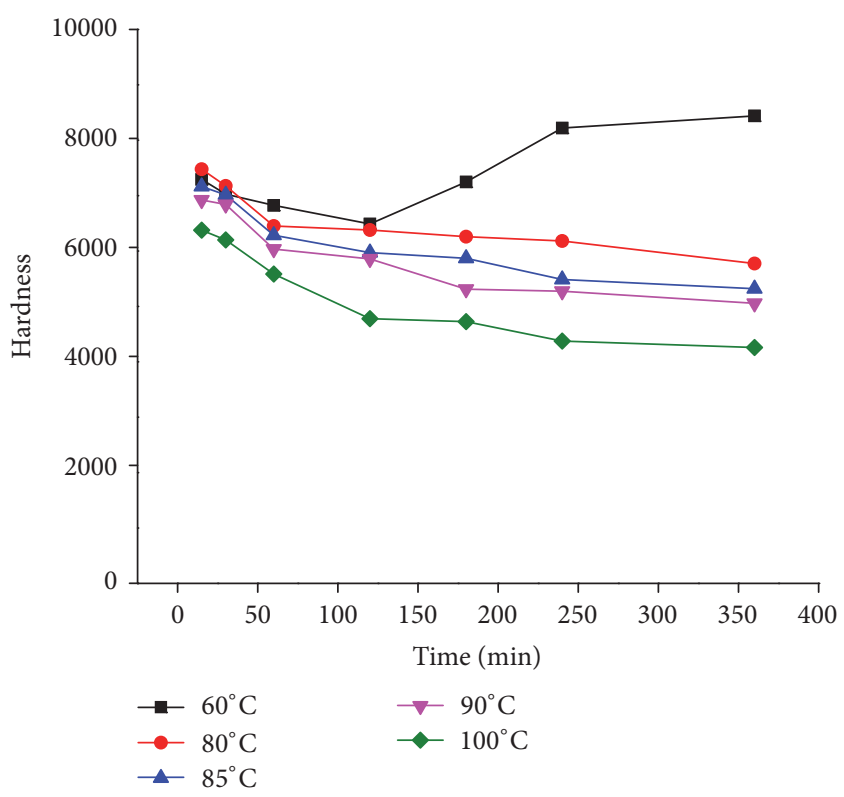

FIgURE 4: Hardness changes during thermal processing.

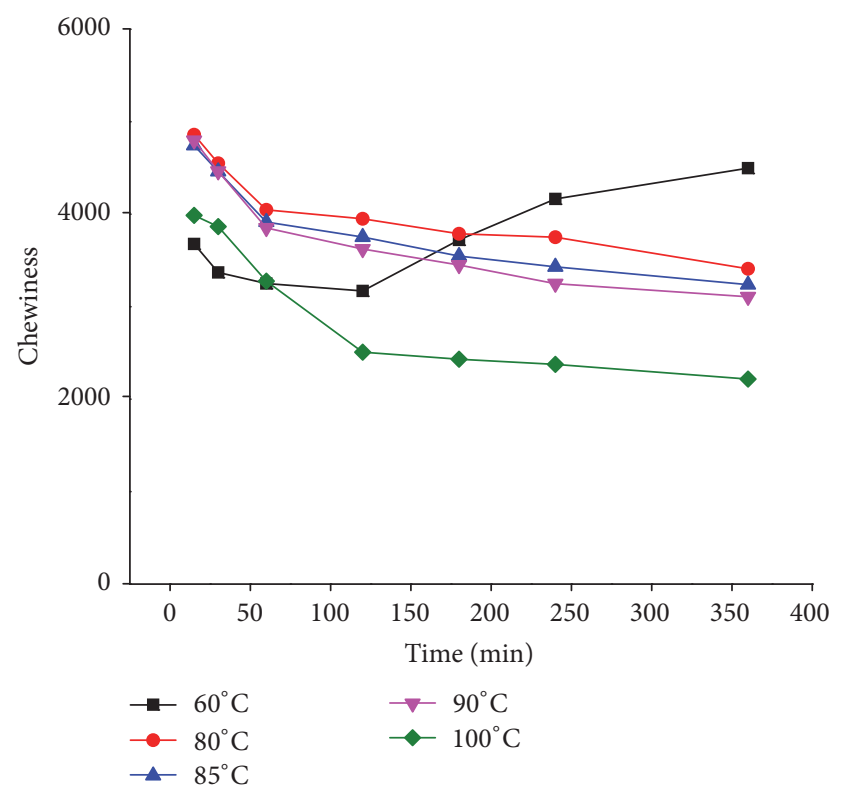

FIGURE 5: Chewiness changes during thermal processing.

3.1.3. Effects on Chewiness. The change in the chewiness was similar to that of the hardness, as shown in Figure 5. The chewiness continued to decrease after cooking at $80,85,90$, and $100^{\circ} \mathrm{C}$ before $120 \mathrm{~min}$, with the most obvious change at $100^{\circ} \mathrm{C}$. Although the chewiness decreased slowly at $60^{\circ} \mathrm{C}$ before $120 \mathrm{~min}$, it then increased rapidly as the time was extended until reaching the maximum. Another finding was that the higher the heating temperature, the more obvious the tendency for the chewiness to be reduced. A slow reduction in chewiness was observed in abalone meat treated at 80,85 , 90 , and $100^{\circ} \mathrm{C}$ from 120 to $360 \mathrm{~min}$. 


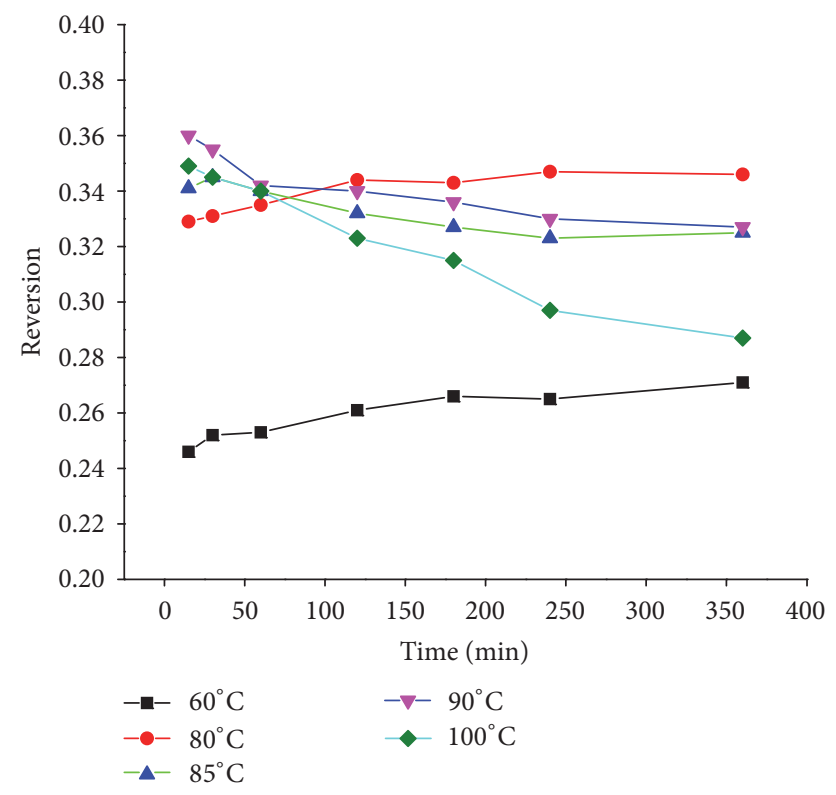

FIGURE 6: Reversion changes during thermal processing.

3.1.4. Effects on Reversion. The change in the reversion over time under different heating conditions is shown in Figure 6. The minimum value of reversion was observed at $60^{\circ} \mathrm{C}$; it then increased gradually at higher temperatures. The reversion continued to reduce after cooking at 85 and $90^{\circ} \mathrm{C}$ but decreased significantly at $100^{\circ} \mathrm{C}$. A slow increase was observed at $80^{\circ} \mathrm{C}$ and it continued to rise slightly until $120 \mathrm{~min}$ had elapsed.

3.1.5. Effects of Low Temperature Heating. As can be seen from the diagram, compared with the higher heating temperature, the springiness, hardness, chewiness, and reversion tended to be abnormal at $60^{\circ} \mathrm{C}$. The abnormal phenomena may be explained by its microstructure and chemical forces. Although the samples' pores became smaller at $60^{\circ} \mathrm{C}$, their network structure becomes very loose compared to unprocessed abalone, resulting in low hardness, springiness, reversion, and chewiness. When the heating time reached $120 \mathrm{~min}$, the pore structure of the network continues to become smaller and the network structure becomes a honeycomb $[14,15]$. The tighter network structure of the honeycomb can increase the abalone's springiness, hardness, chewiness, and reversion.

As the heating time was gradually extended, the chemical forces between protein molecules were destroyed. The mercapto group was exposed to oxidization by disulfide bonds, resulting in complete denaturation and aggregation of the protein, which formed a newly ordered 3-dimensional network structure wherein springiness, hardness, chewiness, and reversion moved towards stability [14].

\subsection{Sensory Fuzzy Comprehensive Evaluation}

3.2.1. Establishment of Fuzzy Evaluation Matrix. 11 selected persons from the sensory evaluation team evaluated the springiness, hardness, and smell of the abalone meat samples.
Taking sample 1 as an example, the distribution of votes is shown in Table 5. After normalizing the evaluation results, the corresponding fuzzy matrix $R_{1}$ was observed. The fuzzy matrix of sample 1 is shown below.

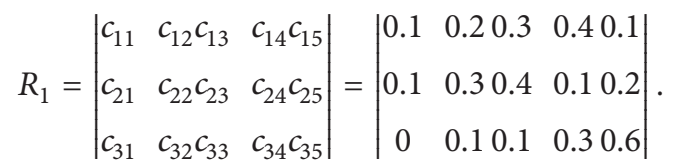

The fuzzy matrices of the other samples could be obtained by the same method, as shown below.

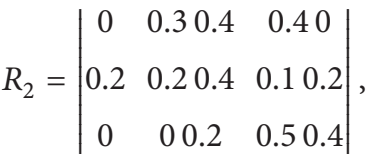

$$
\begin{aligned}
& R_{3}=\left|\begin{array}{ccccc}
0.1 & 0.4 & 0.4 & 0.4 & 0 \\
0.4 & 0.2 & 0.4 & 0.1 & 0.2 \\
0 & 0 & 0.2 & 0.5 & 0.4
\end{array}\right|
\end{aligned}
$$

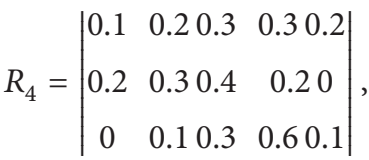

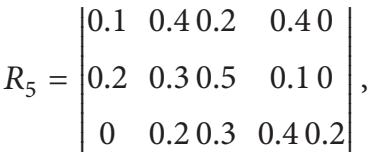$$
R_{6}=\left|\begin{array}{ccccc}
0.2 & 0.1 & 0.3 & 0.3 & 0.2 \\
0.4 & 0.1 & 0.3 & 0.2 & 0.1 \\
0 & 0.1 & 0.4 & 0.4 & 0.2
\end{array}\right|
$$$$
R_{7}=\left|\begin{array}{lllll}
0.2 & 0.2 & 0.3 & 0.3 & 0.1 \\
0.2 & 0.1 & 0.5 & 0.2 & 0.1 \\
0.1 & 0.1 & 0.2 & 0.4 & 0.3
\end{array}\right|
$$

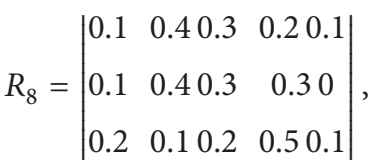$$
R_{9}=\left|\begin{array}{lllll}
0.3 & 0.4 & 0.2 & 0.2 & 0 \\
0.4 & 0.3 & 0.2 & 0.2 & 0 \\
0.4 & 0.4 & 0.2 & 0 & 0.1
\end{array}\right|,
$$$$
R_{10}=\left|\begin{array}{lllll}
0.1 & 0.5 & 0.3 & 0.1 & 0.1 \\
0.4 & 0.1 & 0.3 & 0.2 & 0.1 \\
0.1 & 0.5 & 0.2 & 0.3 & 0.1
\end{array}\right|,
$$

$$
R_{11}=\left|\begin{array}{ccccc}
0.1 & 0.5 & 0.3 & 0.2 & 0 \\
0.4 & 0.2 & 0.3 & 0.1 & 0.1 \\
0.5 & 0.3 & 0.1 & 0.1 & 0.1
\end{array}\right|,
$$




$$
\begin{aligned}
& R_{12}=\left|\begin{array}{ccccc}
0.1 & 0.3 & 0.3 & 0.2 & 0 \\
0.3 & 0.2 & 0.3 & 0.1 & 0.1 \\
0.1 & 0.1 & 0.1 & 0.1 & 0.1
\end{array}\right|,
\end{aligned}
$$

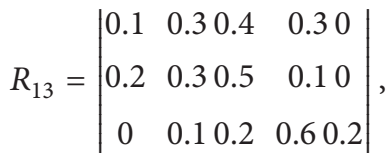$$
R_{14}=\left|\begin{array}{ccccc}
0.1 & 0.5 & 0.2 & 0.3 & 0 \\
0.2 & 0.7 & 0.1 & 0.1 & 0 \\
0 & 0.2 & 0.4 & 0.5 & 0
\end{array}\right| \text {, }
$$$$
R_{15}=\left|\begin{array}{ccccc}
0.3 & 0.3 & 0.4 & 0.1 & 0 \\
0.4 & 0.3 & 0.3 & 0.1 & 0 \\
0.4 & 0.4 & 0.3 & 0 & 0
\end{array}\right|,
$$$$
R_{16}=\left|\begin{array}{lllll}
0.2 & 0.3 & 0.3 & 0.1 & 0.2 \\
0.5 & 0.3 & 0.2 & 0.1 & 0 \\
0.1 & 0.5 & 0.3 & 0.2 & 0
\end{array}\right| \text {, }
$$

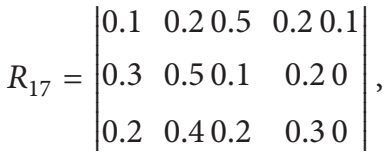

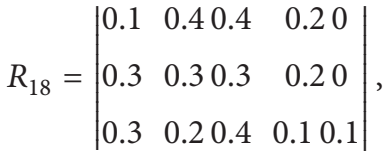$$
R_{19}=\left|\begin{array}{lllll}
0.1 & 0.4 & 0.4 & 0.1 & 0.1 \\
0.5 & 0.1 & 0.3 & 0.1 & 0.1 \\
0.1 & 0.1 & 0.2 & 0.5 & 0.2
\end{array}\right|,
$$

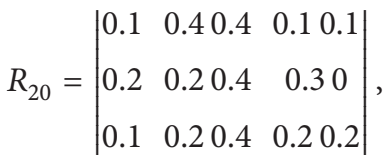

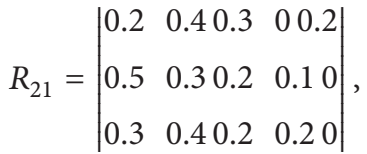$$
R_{22}=\left|\begin{array}{lllll}
0.1 & 0.4 & 0.2 & 0.3 & 0.1 \\
0.4 & 0.2 & 0.3 & 0 & 0.2 \\
0.1 & 0.3 & 0.4 & 0.2 & 0.1
\end{array}\right| \text {, }
$$$$
R_{23}=\left|\begin{array}{lllll}
0.1 & 0.3 & 0.2 & 0.4 & 0.1 \\
0.4 & 0.3 & 0.1 & 0.2 & 0.1 \\
0.5 & 0.3 & 0.1 & 0.2 & 0.1
\end{array}\right|,
$$$$
R_{24}=\left|\begin{array}{ccccc}
0.2 & 0.1 & 0.2 & 0.4 & 0.2 \\
0.3 & 0.1 & 0.4 & 0 & 0.2 \\
0.1 & 0.1 & 0.2 & 0.5 & 0.1
\end{array}\right| \text {, }
$$

TABLE 3: Membership grades of different evaluation indices.

\begin{tabular}{lccc}
\hline & $u_{1}$ & $u_{2}$ & $u_{3}$ \\
\hline Expert 1 & 0.53 & 0.51 & 0.27 \\
Expert 2 & 0.46 & 0.41 & 0.32 \\
Expert 3 & 0.39 & 0.49 & 0.28 \\
Expert 4 & 0.54 & 0.37 & 0.33 \\
Expert 5 & 0.47 & 0.44 & 0.29 \\
\hline
\end{tabular}

TABLE 4: Normalization results of the membership grade.

\begin{tabular}{lccc}
\hline & $u_{1}$ & $u_{2}$ & $u_{3}$ \\
\hline Expert 1 & 0.405 & 0.389 & 0.206 \\
Expert 2 & 0.387 & 0.345 & 0.268 \\
Expert 3 & 0.336 & 0.422 & 0.241 \\
Expert 4 & 0.435 & 0.298 & 0.267 \\
Expert 5 & 0.392 & 0.366 & 0.242 \\
\hline Average value & 0.391 & 0.364 & 0.245 \\
\hline
\end{tabular}

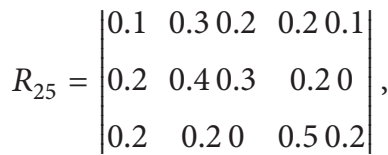

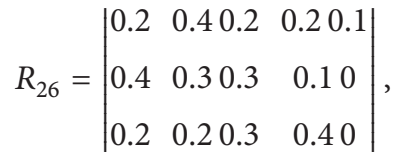

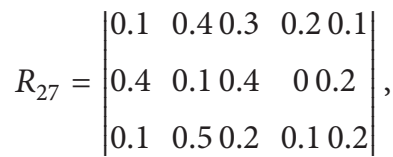

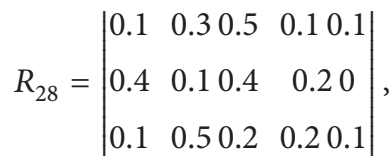

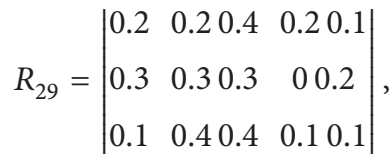

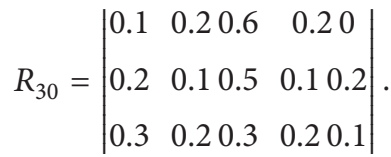

3.2.2. Fuzzy Weight Vector of Evaluation Index. The membership grades of different evaluation indices were given by professional teachers, as shown in Table 3. The normalized results are shown in Table 4.

As can be seen from Table 4 , the weight vector in the fuzzy comprehensive evaluation was $a=(0.391,0.364,0.245)$. 
TABLE 5: Vote distributions of sensory evaluation of sample 1.

\begin{tabular}{|c|c|c|c|c|c|}
\hline \multirow{2}{*}{ Index set } & \multicolumn{5}{|c|}{ Evaluation set } \\
\hline & $v_{1}$ & $v_{2}$ & $v_{3}$ & $v_{4}$ & $v_{5}$ \\
\hline$\overline{u_{1}}$ & $1\left(c_{11}\right)$ & $2\left(c_{12}\right)$ & $3\left(c_{13}\right)$ & $4\left(c_{14}\right)$ & $1\left(c_{15}\right)$ \\
\hline$u_{2}$ & $1\left(c_{21}\right)$ & $3\left(c_{22}\right)$ & $4\left(c_{23}\right)$ & $1\left(c_{24}\right)$ & $2\left(c_{25}\right)$ \\
\hline$u_{3}$ & $0\left(c_{33}\right)$ & $1\left(c_{32}\right)$ & $1\left(c_{33}\right)$ & $3\left(c_{34}\right)$ & $6\left(c_{35}\right)$ \\
\hline
\end{tabular}

Note. $C_{i j}(i=1,2,3 ; j=1,2,3,4,5)$ was the number of votes for the $i$ factor as the $j$ grade. Springiness was $u_{1}$, hardness was $u_{2}$, and smell was $u_{3}$.

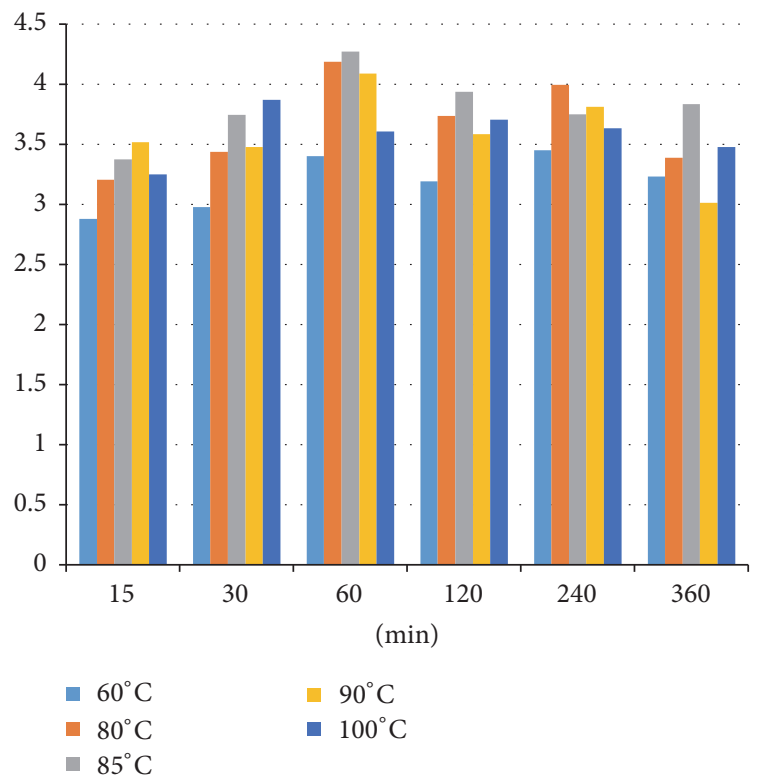

FIGURE 7: Comprehensive evaluation for the scores of abalone meat.

3.2.3. Fuzzy Transformation and Comprehensive Evaluation Results. The comprehensive evaluation results for sample 1 are as follows:

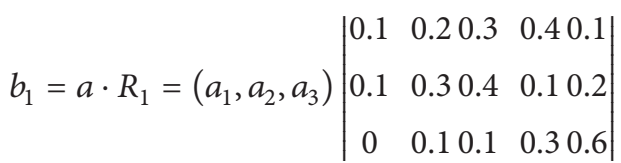

$$
\begin{aligned}
& =(0.391,0.364,0.245)\left|\begin{array}{ccccc}
0.1 & 0.2 & 0.3 & 0.4 & 0.1 \\
0.1 & 0.3 & 0.4 & 0.1 & 0.2 \\
0 & 0.1 & 0.1 & 0.3 & 0.6
\end{array}\right| \\
& =(0.0755,0.2119,0.2874,0.2663,0.2589) \text {. }
\end{aligned}
$$

The results for the other samples are shown in Table 6 .

Furthermore, we could attain sensory scores $(A)$ corresponding to different heating conditions. The higher the sensory score, the higher the quality level. Specifically, a score of $2 \sim 3$ was poor, 3 4 was average, and $4 \sim 5$ was excellent. The results are shown in Figure 7.

Although the sensory evaluation scores for samples 9, 15 , and 18 were, respectively, 4.19, 4.27, and 4.09, which are extremely excellent, most samples scored at the general level. However, sample 1 was the worst. Therefore, sample 9 can
TABLE 6: Fuzzy synthesis evaluation result.

\begin{tabular}{ll}
\hline SN & Fuzzy synthesis evaluation result \\
\hline 1 & $b 1=(0.0755,0.2119,0.2874,0.2663,0.2589)$ \\
2 & $b 2=(0.0728,0.1901,0.351,0.3153,0.1708)$ \\
3 & $b 3=(0.1847,0.2537,0.2755,0.2517,0.1344)$ \\
4 & $b 4=(0.1119,0.2119,0.3364,0.3371,0.1027)$ \\
5 & $b 5=(0.1119,0.3146,0.3337,0.2908,0.049)$ \\
6 & $b 6=(0.2238,0.1,0.3245,0.2881,0.1636)$ \\
7 & $b 7=(0.1755,0.1391,0.3483,0.2881,0.149)$ \\
8 & $b 8=(0.1245,0.3265,0.2755,0.3099,0.0636)$ \\
9 & $b 9=(0.3609,0.3636,0.2,0.151,0.0245)$ \\
10 & $b 10=(0.2092,0.3544,0.2755,0.1854,0.0755)$ \\
11 & $b 11=(0.3072,0.3418,0.251,0.1391,0.0609)$ \\
12 & $b 12=(0.1728,0.2146,0.3391,0.2735,0.1)$ \\
13 & $b 13=(0.1119,0.251,0.3874,0.3007,0.049)$ \\
14 & $b 14=(0.1119,0.4993,0.2126,0.2762,0)$ \\
15 & $b 15=(0.3609,0.3245,0.3391,0.0755,0)$ \\
16 & $b 16=(0.2847,0.349,0.2636,0.1245,0.0782)$ \\
17 & $b 17=(0.1973,0.3582,0.2809,0.2245,0.0391)$ \\
18 & $b 18=(0.2218,0.3146,0.3636,0.1755,0.0245)$ \\
19 & $b 19=(0.2456,0.2173,0.3146,0.198,0.1245)$ \\
20 & $b 20=(0.1364,0.2782,0.4,0.1973,0.0881)$ \\
21 & $b 21=(0.3337,0.3636,0.2391,0.0854,0.0782)$ \\
22 & $b 22=(0.2092,0.3027,0.2854,0.1663,0.1364)$ \\
23 & $b 23=(0.3027,0.3,0.1391,0.2782,0.1)$ \\
24 & $b 24=(0.2119,0.1,0.2728,0.2789,0.1755)$ \\
26 & $b 25=(0.1609,0.3119,0.1874,0.2735,0.0881)$ \\
27 & $b 26=(0.2728,0.3146,0.2609,0.2126,0.0391)$ \\
29 & $b 27=(0.2092,0.3153,0.3119,0.1027,0.1609)$ \\
& $b 28=(0.2092,0.2762,0.3901,0.1609,0.0636)$ \\
28 & $b 29=(0.2119,0.2854,0.3636,0.1027,0.1364)$ \\
& $b 30.1854,0.1636,0.4901,0.1636,0.0973)$ \\
\hline & $b$
\end{tabular}

be determined optimally, showing $85^{\circ} \mathrm{C}$ for 1 hour, which represents the optimal processing scheme.

\subsection{Analysis of Predictive Texture Model}

3.3.1. Determining the Hidden Layer Nodes. Although the number of neurons in the hidden layer has a great impact on the accuracy of the prediction model, difficulties are inherent in determining the numbers of hidden neurons. Through the experimental comparison of neural network prediction 
TABLE 7: Comparison of BP neural network model predictions with actual results.

\begin{tabular}{|c|c|c|c|c|}
\hline $\mathrm{S} / \mathrm{N}$ & Sensory index & Sensory results & Predictive results & Error $/ \%$ \\
\hline \multirow{3}{*}{1} & Springiness & 1.93 & 1.85 & 4.15 \\
\hline & Hardness & 2.38 & 2.61 & 9.66 \\
\hline & Smell & 4.21 & 3.84 & 8.79 \\
\hline \multirow{3}{*}{2} & Springiness & 2.83 & 2.72 & 3.89 \\
\hline & Hardness & 3.29 & 3.16 & 3.95 \\
\hline & Smell & 3.07 & 2.79 & 9.12 \\
\hline \multirow{3}{*}{3} & Springiness & 2.43 & 2.27 & 6.58 \\
\hline & Hardness & 2.79 & 2.97 & 6.45 \\
\hline & Smell & 1.66 & 1.47 & 11.45 \\
\hline \multirow{3}{*}{4} & Springiness & 2.82 & 2.57 & 8.87 \\
\hline & Hardness & 3.4 & 3.21 & 5.59 \\
\hline & Smell & 2.94 & 2.65 & 9.86 \\
\hline \multirow{3}{*}{5} & Springiness & 2.38 & 2.12 & 10.92 \\
\hline & Hardness & 2.32 & 2.29 & 1.29 \\
\hline & Smell & 3.21 & 3.4 & 5.92 \\
\hline \multirow{3}{*}{6} & Springiness & 2.97 & 2.79 & 6.06 \\
\hline & Hardness & 2.25 & 2.16 & 4.00 \\
\hline & Smell & 2.67 & 2.77 & 3.75 \\
\hline \multirow{3}{*}{7} & Springiness & 4.11 & 3.84 & 6.57 \\
\hline & Hardness & 2.67 & 2.27 & 14.98 \\
\hline & Smell & 1.79 & 2.02 & 12.85 \\
\hline \multirow{3}{*}{8} & Springiness & 2.76 & 2.61 & 5.43 \\
\hline & Hardness & 3.2 & 3.01 & 5.94 \\
\hline & Smell & 2.11 & 2.32 & 9.95 \\
\hline \multirow{3}{*}{9} & Springiness & 4.73 & 4.38 & 7.40 \\
\hline & Hardness & 2.55 & 2.74 & 7.45 \\
\hline & Smell & 3.73 & 3.86 & 3.49 \\
\hline \multirow{3}{*}{10} & Springiness & 2.83 & 2.71 & 4.24 \\
\hline & Hardness & 2.24 & 2.39 & 6.70 \\
\hline & Smell & 4.41 & 3.92 & 11.11 \\
\hline \multirow{3}{*}{11} & Springiness & 3.21 & 3.28 & 2.18 \\
\hline & Hardness & 1.82 & 1.78 & 2.20 \\
\hline & Smell & 2.53 & 2.21 & 12.65 \\
\hline \multirow{3}{*}{12} & Springiness & 2.24 & 2.19 & 2.23 \\
\hline & Hardness & 3.85 & 3.74 & 2.86 \\
\hline & Smell & 1.19 & 1.32 & 10.92 \\
\hline Average error/\% & & & & 6.93 \\
\hline
\end{tabular}

results, it can be shown that the number of hidden layer nodes was 17.

3.3.2. Validation of Predictive Texture Model. The BP neural network was used to predict the sensory evaluation data of abalone meat. The results show little difference between the predicted and measured values, with the average error of calculation being relatively small. The prediction results varied for different samples, as did those for different texture parameters of the same sample.

Based on the data in Table 7, the maximum error was $14.98 \%$, the minimum was $1.29 \%$, and the average was $6.93 \%$. These results demonstrate that the established BP neural network has a good prediction effect and the advantages of high prediction efficiency. Thus TPA texture parameters can be used to predict human sensory evaluation results via the BP neural network model.

\section{Conclusion}

(1) The texture and sensory characteristics of abalone meat were analyzed under different heat treatments. A significant decline in hardness occurred at a shorter heat time ( $<60 \mathrm{~min})$, which may have increased the sensory score. Heat treatment for an extended period (>120 min), meanwhile, resulted in no significant change in the hardness and sensory score. The 
heat treatment time $(<120 \mathrm{~min})$ potentially led to a significant decline in springiness and chewiness. Thereafter, a longer heating time (>120 min) had little effect on springiness and chewiness, which tended to be stable. An appropriate increase in the heating temperature might result in a lower level of hardness and chewiness; a significantly low level of hardness, springiness, and chewiness was observed in boiled samples. The increase and decrease of heating time were associated with the change of chewiness to a very small degree. Moreover, a low level of chewiness could be obtained at $60^{\circ} \mathrm{C}$. The heating condition of $60^{\circ} \mathrm{C}$ was abnormal compared to the higher temperature heating conditions, which could have been a result of changes in chemical forces.

(2) A sensory evaluation of abalone meat was conducted using fuzzy mathematics, which is beneficial for avoiding interference from subjective factors and obtaining fair results. A suitable temperature and time for the preparation of abalone meat should be around $85^{\circ} \mathrm{C}$ for $60 \mathrm{~min}$, at which condition the evaluation score was 4.27 , higher than at other temperatures and times; the springiness and resilience were maintained at a high level, and the hardness and chewiness were moderate.

(3) The method of using the BP neural network was introduced to predict sensory evaluation parameters of sensory hardness, springiness, and smell, based on the TPA parameters of the texture analyzer test. The results showed that the average error of the prediction model could reach $6.93 \%$. To sum up, this method offers great advantages and application prospects because of its simplicity, handling convenience, and high accuracy.

\section{Conflicts of Interest}

The authors declare that there are no conflicts of interest regarding the publication of this paper.

\section{Acknowledgments}

The authors acknowledge the National Engineering Research Center of Seafood. This work was supported by Public Science and Technology Research Funds Projects of Ocean (no. 201505029).

\section{References}

[1] B. W. Zhu, Research on the Theory and Technology of Seafood Processing, Beijing Science Press, 2010.

[2] W. Ying et al., "Analysis of trace elements in abalone and sea cucumber," Spectroscopy and Spectral Analysis, vol. 29, no. 2, pp. 511-514, 2009.

[3] L. Gao, Texture Changes of Abalone Muscle under Different Processing Conditions, Chinese Marine University, 2011.

[4] G. H. Xiao et al., "Effects of hot processing conditions on partial processing properties of abalone," Journal of Dalian Institute of Light Industry, vol. 31, no. 1, pp. 1-7, 2012.

[5] Z. L. Sun et al., "Design and experiment of bionic food texture analyzer," Transactions of the Chinese Society for Agricultural Machinery, vol. 43, no. 1, pp. 230-234, 2012.

[6] A. M. Peres, L. G. Dias, T. P. Barcelos, J. Sá Morais, and A. A. S. C. Machado, "An electronic tongue for juice level evaluation in non-alcoholic beverages," in Proceedings of the Eurosensors 23rd Conference, pp. 1023-1026, September 2009.

[7] B. Zhu, X. Dong, L. Sun et al., "Effect of thermal treatment on the texture and microstructure of abalone muscle (Haliotis discus)," Food Science and Biotechnology, vol. 20, no. 6, pp. 14671473, 2011.

[8] R. H. Wang et al., "Fuzzy Comprehensive Evaluation Method to Optimize the Production Process with Braised Pork in Brown Sauce," Science and Technology of Food Industry, pp. 274-278, 2015.

[9] G. Y. Sanchez-Brambila, B. G. Lyon, Y. W. Huang, C. E. Lyon, and K. W. Gates, "Sensory characteristics and instrumental texture attributes of abalones, Haliotis fulgens and cracherodii," Journal of Food Science, vol. 67, no. 3, pp. 1233-1239, 2002.

[10] S. Zhu et al., Study on the Processing Technology of Abalone, University of Agriculture and Forestry In Fujian, 2013.

[11] Y. W. Wu, Experiment Design and Data Processing, Suzhou University Press, 2002.

[12] W. Wei, Principles and Applications of Artificial Neural Networks, Beijing University of Aeronautics and Astronautics Press, 1995.

[13] X. D. Wang et al., "Classifying beef muscle and fat pixels using bp-ga neural network," Transactions of the Chinese Society of Agricultural Engineering, pp. 216-219, 2007.

[14] L. Deng, Y. Li, X. Dong, Y. Wang, Q. Xin, and J. Pan, "Chemical interactions and textural characteristics of abalone pleopod muscle protein during heating," Transactions of the Chinese Society of Agricultural Engineering, vol. 30, no. 18, pp. 307-316, 2014.

[15] Y. Q. Zhang et al., "Comparative study on natural drying and hot-air drying of abalone," Transactions of the CSAE, vol. 24, no. 1, pp. 296-299, 2008. 

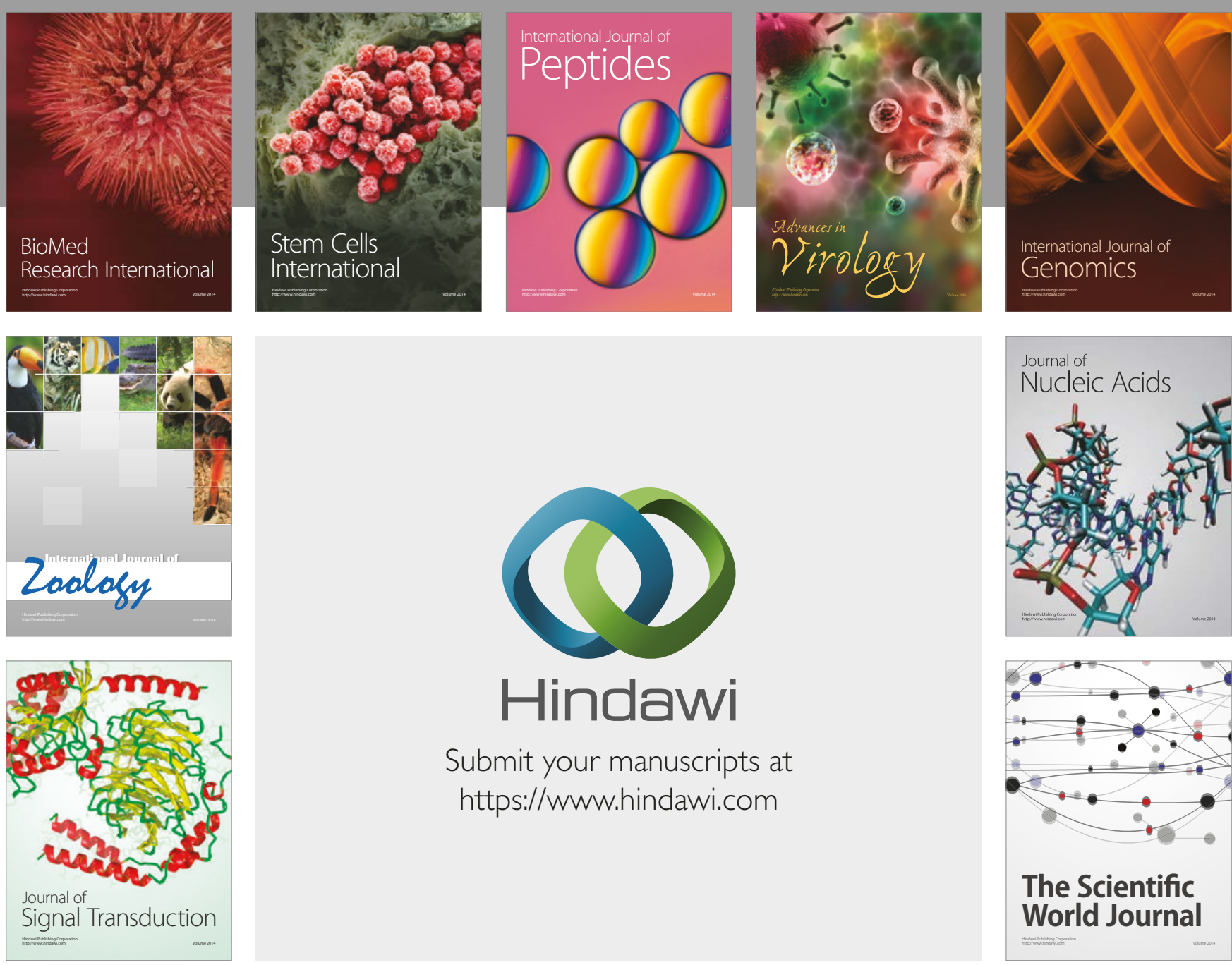

Submit your manuscripts at

https://www.hindawi.com
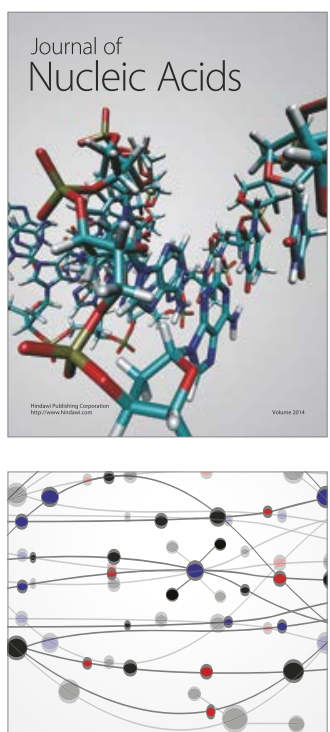

The Scientific World Journal

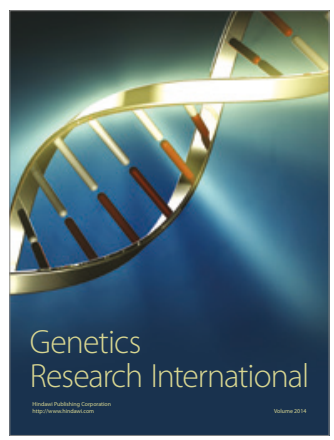

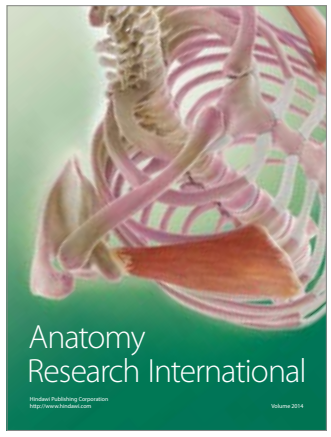

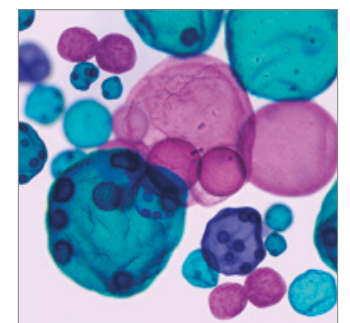

International Journal of Microbiology
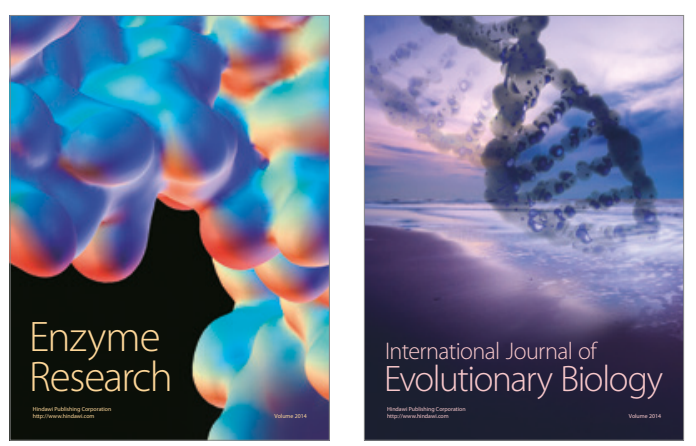
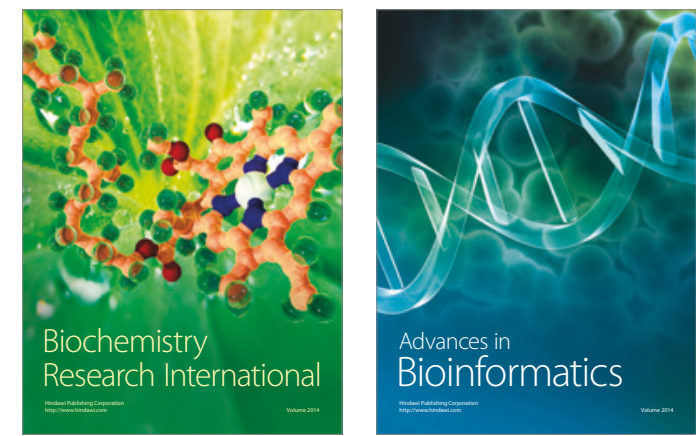

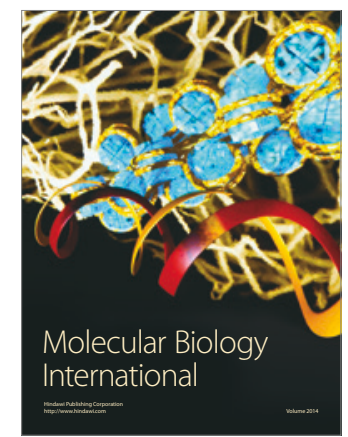

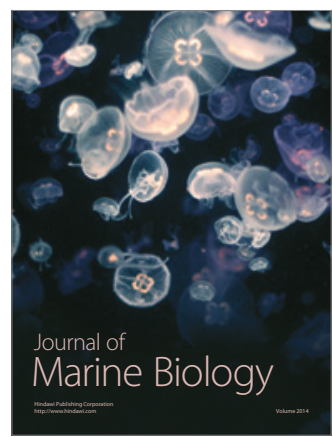

